\title{
TESELACIONES COMO ESTRATEGIA DIDÁCTICA PARA EL APRENDIZAJE DE LA SIMETRÍA
}

\author{
Lucía Solís-Castro, Socorro Daly-Soto \\ Universidad Nacional. (Costa Rica) \\ lucydango@hotmail.com,socorrodaly@gmail.com
}

\section{Resumen}

El presente artículo es un reporte de un taller realizado en la RELME 31. Para la contextualización del contenido del taller se inició con una breve reseña histórica de los orígenes de las teselaciones, usos, desarrollo teórico, diferencia entre teselación y tesela y su principal exponente. Posteriormente, se implementan tres actividades didácticas. La primera consta en la reproducción de una teselación en una hoja tipo cuadricula dada. Para la segunda actividad, a partir de una tesela deben encontrar un patrón para cubrir una superficie plana dada en su totalidad. Para la última actividad, con un círculo de papel y un patrón dado, crean un copo de nieve aplicando simetría.

Palabras clave: tesela, teselación, didáctica, matemática, simetría.

\section{Abstract}

This paper is a report of a workshop held at the 31st RELME. For the contextualization of the workshop content, we started with a brief historical review of the origins, uses, and theoretical development of tessellations, as well as the difference between tile and tessellation, and its main exponent. Subsequently, three didactic activities were implemented. The first one consists in the reproduction of a tessellation in a given grid sheet. For the second activity, from a tile, they must find a pattern to cover a given flat surface in its entirety. For the last activity, with a circle made of paper and a given pattern, they must create a snowflake by applying symmetry.

Key Words: tile,_tessellation, didactics, math, symmetry.

\section{Introducción}

El estudio de simetrías forma parte de los currículos matemáticos de la región en diferentes niveles del sistema educativo, por lo tanto, toma relevancia la incorporación de nuevas formas de enseñanza a través de actividades educativas para dejar de lado la monotonía de las lecciones clásicas de matemática procurando mayor interés por parte de la comunidad estudiantil.

El taller es dirigido a docentes del área de matemáticas de todos los niveles de la región. El mismo, se crea con el propósito de profundizar conceptos de la teoría de simetrías saliendo de forma magistral tradicional de dar los conceptos. A través de tres actividades didácticas independientes, se busca estimular la capacidad de abstracción en los participantes del taller. 
Ahora, se considera la definición de teselados de Uribe Garzón, S. M., Cárdenas Forero, Ó. L., \& Becerra Martínez, J. F. (2014) son "creaciones artísticas que, repetidas sobre el plano, llenan completamente una región sin vacíos ni superpuesto" las mismas se plantean como herramientas de enseñanza.

Se parte con la pregunta de investigación ¿en qué medida se estimula la capacidad de abstracción utilizando teselaciones como estrategia didáctica para el aprendizaje de la simetría?

\section{Marco teórico de la propuesta del taller}

Para poder implementar el uso de las teselaciones en el ambito de la Matemática Educativa, es necesario tener en cuenta algunos conceptos previos, los cuáles, Escher describe y permiten entender sus técnicas, entre estas:

a) Todo aquel que desee representar una figura simétrica sobre una superficie plana, deberá tomar en consideración tres principios cristalográficos fundamentales:

- Traslación: Realiza un desplazamiento paralelo de una figura.

- Rotación: Mueve una figura en torno a un punto fijo de rotación y ángulo.

- Reflexión: Todos los puntos del plano son enviados a sus imágenes reflejadas con respecto a una recta de reflexión que actúa como espejo.

b) Empleo de las figuras como trasfondo: Los ojos están hechos para fijarse en un determinado objeto, el resto del campo visual se convierte en fondo.

c) Desarrollo de formas y contrastes

d) El número infinito: Si se quiere representar un número infinitamente grande, se debe reducir gradualmente el tamaño de las figuras y sugerir así que han alcanzado el límite del tamaño infinitamente pequeño.

e) Estampas que narran una historia: La característica principal es la transición de la superficie plana al espacio tridimensional. (Herrera et al., 2010, p.425)

Sobre el uso de las teselaciones en la enseñanza, Angel, M. P et al. (2008) mencionan:

Las simetrías se reconocen en los mosaicos por la presencia de figuras divididas en dos mitades iguales. Así pues, siempre van a ser los elementos ópticos los que se utilicen para reconocer las isometrías y para construir sus primeras concepciones, a partir de las cuales se podrán derivar más adelante las definiciones y propiedades matemáticas. Por lo tanto, es importante hacer énfasis en esos elementos ópticos típicos de cada isometría, pues una introducción gráfica intuitiva a las isometrías podrá llevar después, con muy poco trabajo, a la idea matemática; sin embargo, la introducción de las isometrías a partir de sus definiciones matemáticas no tiene por qué llevar a la idea gráfica. (p.4)

Por lo anterior, el taller tuvo tres objetivos fundamentales: Realizar actividades que permitan profundizar en la noción intuitiva de simetría que son características de las teselas, teselaciones y teselados, reflexionar 
sobre cómo esta noción compleja se puede trabajar en el aula con estudiantes de educación secundaria y mostrar cómo otros contenidos se pueden abordar a partir de las características de las teselaciones algunas nociones matemáticas como áreas, volúmenes, funciones, perímetro y operaciones con fracciones.

\section{Metodología del taller}

Se presentan tres tipos de procedimientos con objetos presentes en el entorno escolar y del hogar donde deben detectar y reproducir algún tipo de teselación. Lo anterior se hace por medio de observaciones y propuestas tipo prueba y error permitiendo conocer aspectos epistemológicos, heurísticos y cognitivos de los participantes. Cada una de las expositoras ejerceré un papel de mediador de los conocimientos y guía a los participantes a través de las distintas actividades. Al finalizar cada actividad se les pide a los participantes describir en el material proporcionado la estrategia desarrollada.

\section{Actividades para el taller}

Actividad introductoria: Para esta actividad el participante debe poseer una hoja de color, tijera y cinta (todo entregado previamente por el equipo del taller). Siguiendo las instrucciones de una de las talleristas va doblar, recortar, rotar, traladar y pegar las partes de la figura hasta crear una tesela. Posteriormente, todo los tarticipantes se trasladan hasta un espacio designado y deben, entre todos, construir un teselado en la superficie del suelo con las teselas creadas mientras las talleristas los les dan seguimiento mediante preguntas generadoras.

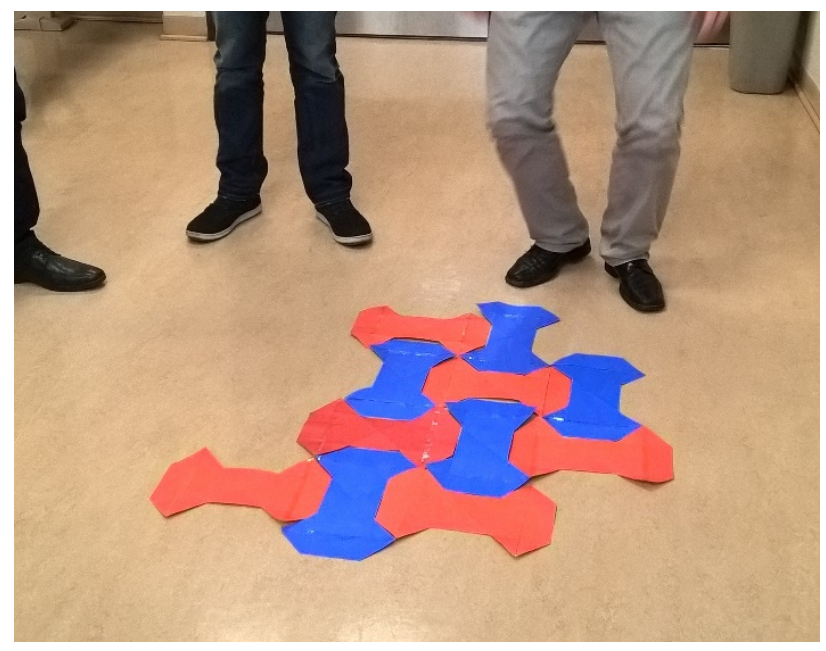

Figura 1. Teselado creado por los participantes del taller. 


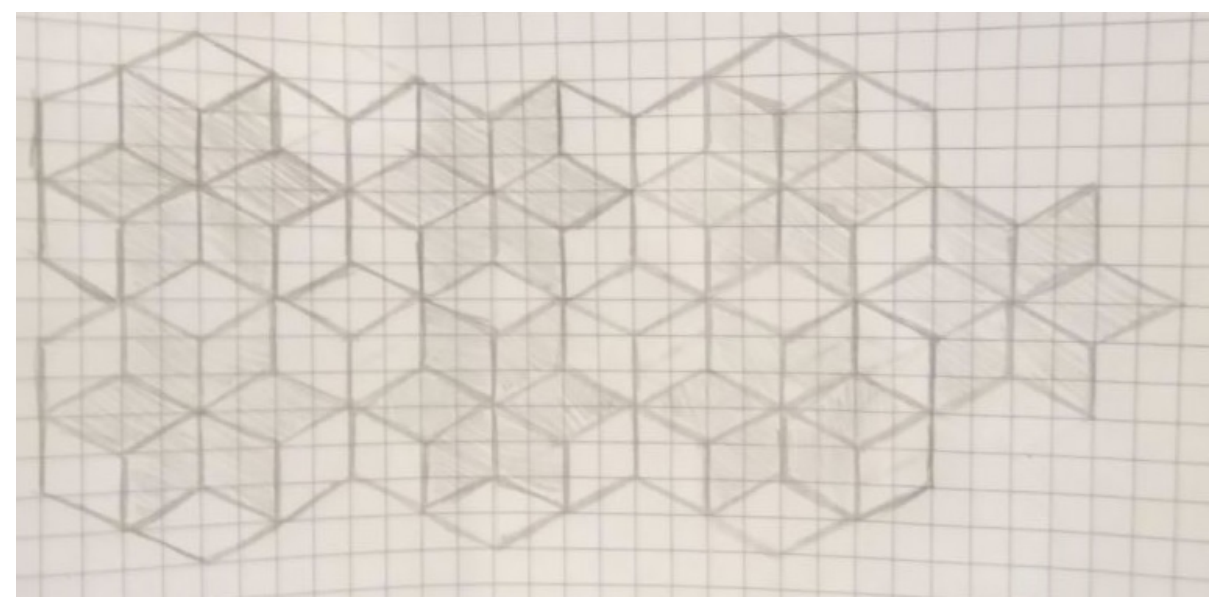

Figura 2. Patrón de teselación

Actividad de percepción: Para esta actividad el participante debe poseer una hoja de cuadricula, una imagen del teselado y un lápiz (todo entregado previamente por el equipo del taller). Debe encontrar el patrón de la teselación e intentar plasmarlo en la hoja de cuadricula. Esta actividad tuvo gran aceptación por parte de los participantes alabando principalmente el esfuerzo y la capacidad de abstracción necesaria para realizarla.

Actividad de reproducción: Para esta actividad el participante debe poseer una hoja punteada, una imagen de tesela y un lápiz (todo entregado previamente por el equipo del taller). Debe encontrar la manera de reproducir la tesela en la hoja punteada hasta completar la hoja en su totalidad. Esta actividad puso a prueba la creatividad de los participantes, ya que, encontraron maneras muy diferentes de realizar la tarea.
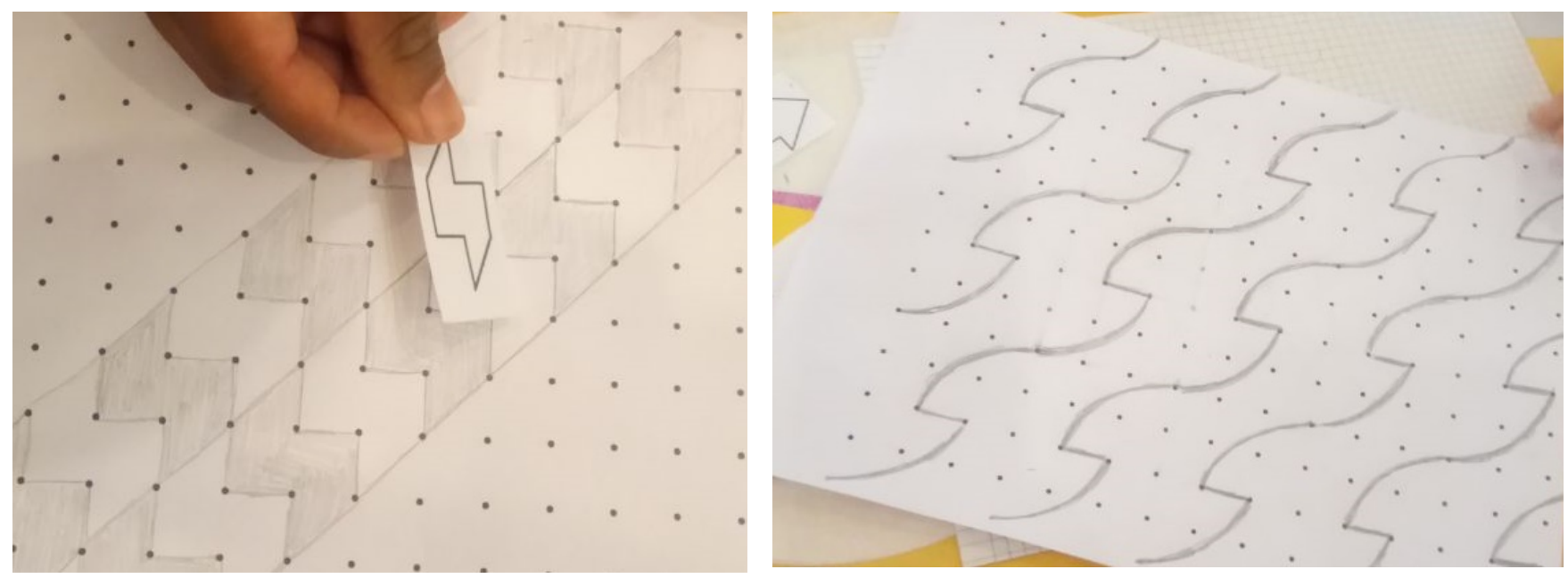

Figura 3. Teselas a reproducir 
Actividad de ejecución: Por último, a cada participante se le otorga un par de círculos de café, un lápiz, una tijera y un patrón. Cada circulo de papel debe ser doblado a la mitad la cantidad de veces propuesta por la tallerista. Posteriormente, deben de reproducir el patrón dado y proceder a recortarlo. Esta actividad es la preferida de los participantes. Los ayuda a comprender con qué facilidad se puede enseñar simetrías de una manera dinámica y activa para los estudiantes.
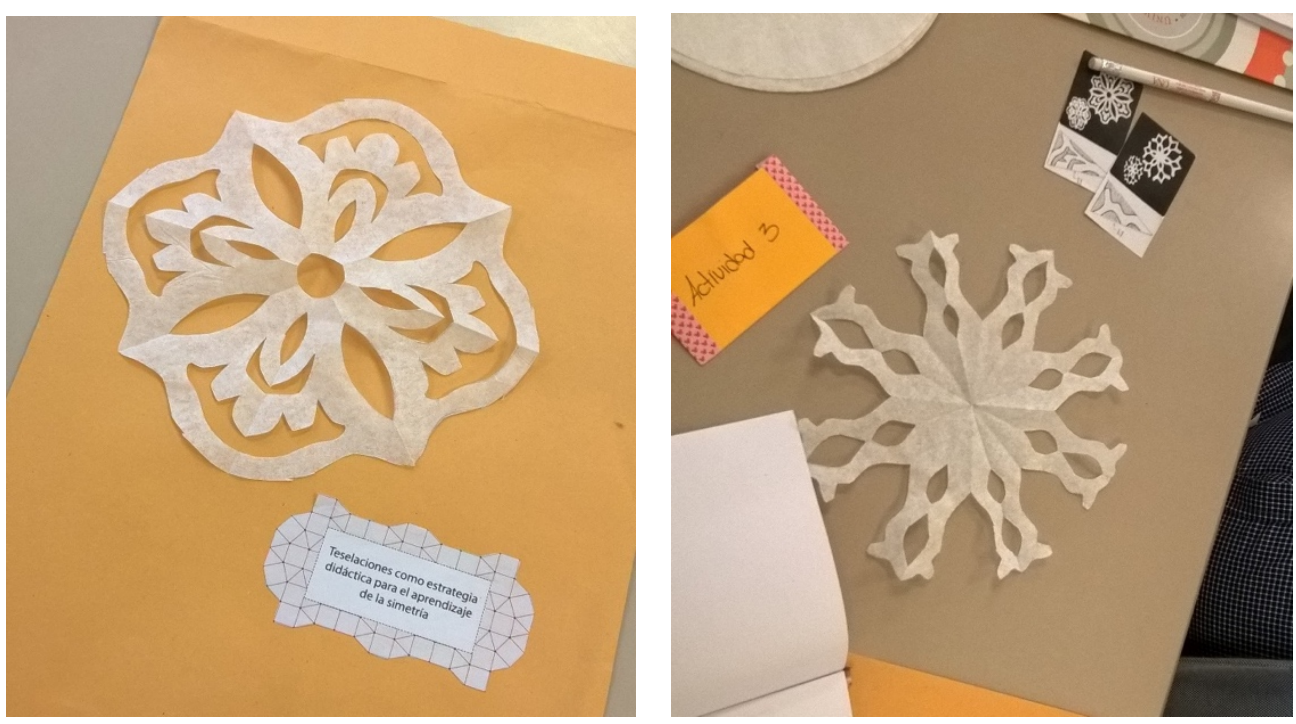

Figura 4. Patrón de teselación

\section{Consideraciones finales}

Se considera que al concluir el taller los docentes participantes cuentan con herramientas para profundizar, de manera sencilla, la noción intuitiva de simetría que son características de las teselas, teselaciones y teselados, para llevarlos al aula con estudiantes de educación secundaria. Se ha podido vivenciar que las características de las teselas permiten trabajar otras nociones matemáticas como áreas, volumes, funciones, perímetro y operaciones con fracciones.

\section{Referencias bibliográficas}

Angel, M. P., Jiménez, W., Rojas, S., Zambrano, N., \& Mora, L. C. (2008). Teselados en el club de Matemáticas. En Luque, Carlos Julio (Ed.), Memorias XVIII Encuentro de Geometria y VI encuentro de Aritmética (pp. 1521). Bogotá, Colombia: Universidad Pedagógica Nacional.

Herrera, V. C., Montes, Y. E., Cruz, A. C., \& Vargas, Á. R. (2010). Teselaciones: Una Propuesta para la Enseñanza y el Aprendizaje de la Geometría a Través del Arte.

Uribe Garzón, S. M., Cárdenas Forero, Ó. L., \& Becerra Martínez, J. F. (2014). Teselaciones para niños: una estrategia para el desarrollo del pensamiento geométrico y espacial de los niños. Educación matemática, 26(2), 135-160. 


\section{CONCEPCIONES QUE SOBRE LA PENDIENTE TIENEN ESTUDIANTES PREUNIVERSITARIOS ANTES DE SU ENSEÑANZA FORMAL}

Martha Iris Rivera López, Crisólogo Dolores Flores

Universidad Autónoma de Guerrero. (México)

caneiris 037@hotmail.com, cdolores2@gmail.com

\section{Resumen}

En este escrito se presentan los avances de una investigación cuyo objetivo es estudiar las concepciones de los estudiantes preuniversitarios acerca de la pendiente, previas a su enseñanza formal. Los datos se obtienen por medio de una entrevista basada en tareas, la cual estuvo integrada por trece tareas que involucraron concepciones de la pendiente ya detectadas por otros investigadores. Para el análisis de los datos se empleará el método de Análisis Temático. El estado actual de la investigación se encuentra en la fase dos del análisis de los datos. Se entrevistaron a 32 estudiantes preuniversitarios (15 a 16 años) que no habían recibido enseñanza formal sobre el concepto de pendiente. Las entrevistas se han transcrito, leído y releído con el objetivo de familiarizarse con los datos y el lenguaje usado por los estudiantes. Se han obtenido las codificaciones iniciales, encontrando que la pendiente para los alumnos es: la recta o diagonal, es una subida o bajada, o una inclinación.

Palabras Clave: concepciones, pendiente, razón de cambio.

\section{Abstract}

This paper shows advances of a research aimed at studying high school students' conceptions on slope, previous to its formal teaching. The data are obtained by means of a task-based interview, which includes thirteen tasks that involved slope conceptions already detected by other researchers. The thematic analysis method is being used for the analysis of data, which is in the second phase at present. Thirty-two high school students (15 to 16 years old), who had not taken formal teaching about the concept of slope, were interviewed. Interviews have been transcribed, read and re-read with the aim to become familiar with the data and language used by the students. The initial encodings have been obtained, finding that for students the slope is: the straight or diagonal line, an ascent or descent, or an inclination.

Key Words: conceptions, slope, rate of change.

\section{Problemática y antecedentes de la Investigación}

La pendiente es una particularidad de la razón de cambio (Stanton y Moore-Russo, 2012), y en México se estudia formalmente en el bachillerato (preuniversitario) en el curso de Matemáticas III (Geometría Analítica). Estos conceptos son importantes debido a su utilidad en la resolución de problemas de 
variación y cambio, ligados a la vida cotidiana y, a su vez son precedentes esenciales en la construcción de la derivada (Dolores, 2013; Stanton y Moore-Russo, 2012; Teuscher y Reys, 2012).

En los programas de Matemáticas de la educación secundaria se sugiere por primera vez en la currícula escolar, la conexión de la razón de cambio con la pendiente (SEP, 2011, p.50). Sin embargo, pareciera que para los estudiantes esto no es así, pues en México como en otros países, las concepciones que tienen acerca de este concepto es limitado o más aún no los ven relacionados entre sí (Moore-Russo, Conner y Rugg, 2011; Nájera, 2015; Stump, 1999; Teuscher y Reys, 2010). Stump (2001) y Walter y Gerson (2007) atribuyen dificultades en la comprensión de este concepto matemático a la diversidad de significados que los profesores asocian a la palabra pendiente (inclinación, declive, empinada, entre otras) y a la escasa o nula conexión que hacen con la razón de cambio. Por otro lado, generalmente su enseñanza se centra en la ejercitación de la fórmula $m=\frac{y_{2}-y_{1}}{x_{2}-x_{1}}$, dejando de lado su interpretación en situaciones de la vida cotidiana, lo cual orilla a su memorización por parte de los alumnos (Wagener, 2009). Además, como razón de cambio tiene poco o ningún énfasis en la interpretación de lo que significa el resultado dentro de una situación dada o sin tener en cuenta las unidades de medida (Herbert y Pierce, 2008). Esta situación implica una débil transición entre la pendiente como cálculo donde pesa más lo algorítmico a su significado como medición de la variación a través de la razón de cambio en contextos ligados a la vida cotidiana (Walter y Gerson, 2007; Planinic, Milin-Sipus, Katic, Susac y Ivanjek, 2012; Nájera, 2015; Wilhelm y Confrey, 2003).

Con el objetivo de contribuir a la mejora en comprensión de la pendiente, los investigadores se han interesado en detectar primeramente las concepciones que están en juego en el proceso de enseñanzaaprendizaje. Stump (1999, 2001) detecta ocho concepciones y Moore-Russo et al. (2011) las extienden, aumentando tres más, teniendo así un total de 11 concepciones; de las cuales podemos identificar que cinco están ligadas a la variación y el cambio (razón geométrica, razón algebraica, propiedad funcional, situaciones del mundo real, indicador de comportamiento) y seis vinculadas a propiedades geométricas (propiedad física, coeficiente paramétrico, concepción trigonométrica, concepción en cálculo, determinador de propiedad y constante de linealidad). Al respecto, Stanton y Moore-Russo (2012) encuentran que los documentos oficiales de secundarias estadounidenses involucran al menos 5 de las 11 concepciones, estas son: razón geométrica, razón algebraica, indicador de comportamiento, determinador de propiedad y propiedad funcional.

Por su parte, Nagle, Moore-Russo, Viglietti y Martin (2013) al comparar las concepciones de la pendiente que tienen los estudiantes de nivel superior con las de profesores del mismo nivel, apreciaron que la concepción como indicador de comportamiento fue sobresaliente en los estudiantes, lo contrario en los profesores. De la misma manera, en los profesores es relevante la concepción de la pendiente en la aplicación a situaciones del mundo real, no así para los estudiantes. Mientras que en los profesores de secundaria, según del estudio de Hoffman (2015), la concepción que predomina en ellos es la razón algebraica seguida de la propiedad física, y es escasa la concepción trigonométrica, en la asociada al cálculo diferencial como derivada, en la determinante de propiedad como paralela o perpendicular y como constante lineal. Con respecto al concepto imagen que tienen los profesores de la pendiente, declaran que este aparece cuando relacionan inmediatamente a una situación del mundo real, como es en rampas, escaleras, techos de casa, calles empinadas, etc. 
Las investigaciones anteriores han dado cuenta de las concepciones que de pendiente tienen los estudiantes (Casey y Nagle, 2016; Stump, 2001), los profesores (Stump, 1999; Hoffman, 2015, Nagle et al., 2013) y las que están presentes en el curriculum estadounidense (Nagle y Moore-Russo, 2014; Stanton y MooreRusso, 2012), sin embargo, hay vacíos y necesidades por satisfacer en esta línea de investigación. Por ejemplo, Stanton y Moore-Russo (2012) plantean la necesidad de investigar las concepciones iniciales de pendiente que tienen los estudiantes de este concepto. Nagle et al. (2013) plantean la búsqueda de la existencia de una relación jerárquica entre concepciones de la pendiente, o bien centrarse en el papel que juegan los profesores de secundaria en el desarrollo de las concepciones que se forman los estudiantes de este nivel. Hoffman (2015) propone un estudio referente a la comparación de las concepciones de la pendiente en los estudiantes de escuela secundaria y la de sus profesores, o una comparación de las concepciones de los profesores de secundaria con profesores de la educación media superior. Por nuestra parte hemos elegido el primero, el que se centra en la investigación de las concepciones iniciales o previas a la enseñanza formal de la pendiente.

El estudio se centra en el bachillerato ya que el estudio de concepciones sobre la pendiente en este nivel educativo es escaso en México. Asimismo la enseñanza de la pendiente o la razón de cambio es formal en este nivel, lo que lleva al estudiante a modificar o enriquecer sus concepciones. Es indiscutible que en el proceso de enseñanza y aprendizaje se tienda a provocar cambios conceptuales en los estudiantes sobre la base del conocimiento de sus ideas previas, por lo que como afirma Ausubel (1978) en el epígrafe de su obra: "Si tuviese que reducir toda la psicología educativa a un solo principio, enunciaría este: El factor más importante que influye en el aprendizaje es lo que el alumno ya sabe. Averígüese esto y enséñese consecuentemente". Es decir, que para mejorar el aprendizaje es necesario conocer los conocimientos precedentes de los estudiantes, particularmente el relacionado con el conocimiento a ser enseñado, porque este tendrá una influencia decisiva en su aprendizaje. Puede convertirse en obstáculo o en punto de partida para que, en el proceso de enseñanza pueda ser trascendido para acercarlo al conocimiento aceptable. Ignorar sus conocimientos precedentes seguramente conducirá a la preservación de concepciones alternativas, preconceptos o conceptos erróneos, que de por sí son difíciles de remover o trascender incluso con la enseñanza reiterada. Por ello este trabajo de investigación se ha planteado la siguiente pregunta de investigación: ¿Qué concepciones tienen los estudiantes acerca de la pendiente antes de ser enseñada formalmente en el bachillerato?

\section{Marco conceptual}

La razón de cambio y la pendiente son considerados como conceptos equivalentes en este trabajo. El primero es utilizado para modelar procesos de variación y cambio, el segundo es un concepto geométrico que describe la inclinación de las rectas o curvas. Se entrecruzan cuando esas rectas o curvas son utilizadas como representaciones gráficas de funciones que modelen situaciones variacionales. Ambos son presentados con el mismo modelo matemático, es decir un cociente de diferencias $\frac{y_{2}-y_{1}}{x_{2}-x_{1}}$. A medida que el concepto de pendiente es utilizado para medir el cambio en situaciones que impliquen una razón de cambio constante o no constante, este suele denominarse razón de cambio, además de que este involucra diversos contextos como la física, la biología, la economía, cálculo, etc. Las concepciones son asumidas en el mismo sentido que Thompson (1992): "son ideas, opiniones o juicios que forman parte del pensamiento. Son una estructura mental general que abarca creencias, significados, conceptos, proposiciones, reglas, imágenes mentales, preferencias y similares (Thompson, 1992, p. 130). 


\section{Metodología}

Participaron 32 estudiantes de bachillerato que aún no han cursado Matemáticas III o no han trabajado formalmente el concepto de pendiente. La recolección de datos se realizó a través de una entrevista basada en tareas que incluyó las concepciones de pendientes que ya han sido categorizadas por otros investigadores (Dolores, Alarcón \& Albarrán, 2002; Moore-Russo et al., 2011; Stump, 1999, 2001). Consideramos que la entrevista basada en tareas es adecuada porque permite observar el comportamiento matemático de las ideas que usan los participantes. Además, esto nos permitirá hacer inferencias sobre el posible significado matemático que se les atribuye (Goldin, 1997). Para nuestro estudio, la entrevista basada en tareas sirve para explorar las concepciones de la pendiente que cada participante tiene o pone en práctica al resolver cada una de las tareas. La entrevista constó de 13 tareas que se dan en diferentes contextos, fue aplicada individualmente y con una duración promedio de una hora, esta fue semiestructurada, ya que se dio la libertad de agregar otras preguntas a fin de profundizar sobre su respuesta o procedimientos.

Para analizar los datos, utilizamos el análisis temático sugerido por Braun y Clarke (2012), este método permite identificar, organizar y ofrecer información sistemática sobre patrones de significado (temas) a través de un conjunto de datos. Este método se estructura en seis fases:

- Familiarizarse con los datos. Se transcriben y leen repetidamente las entrevistas, con el objetivo de familiarizarse con los datos y el lenguaje usado por los estudiantes.

- Generación de códigos iniciales. Sobre la base de la pre-lectura de las narrativas, se establecen códigos iniciales para una primera clasificación. Se buscan palabras o frases utilizadas por los estudiantes para referirse a la pendiente, sus procedimientos y justificaciones.

- Búsqueda de temas. Se revisan los datos codificados para identificar las áreas de similitud y superposición entre los códigos.

- Revisar los temas. Esta fase implica un proceso recursivo mediante el cual se revisan los temas en desarrollo en relación con los datos codificados y el conjunto completo de datos. Esta fase se centra esencialmente en el control de calidad.

- Definir y nombrar temas. Al definir los temas, se necesita ser capaz de expresar claramente lo que es único y específico sobre cada tema, si puede resumir la esencia de cada tema en unas pocas oraciones es una buena prueba de esto.

- Producir el informe. La fase final del análisis es la producción de un informe como un artículo de revista o una disertación. El propósito de su informe es proporcionar una historia convincente sobre sus datos basados en el análisis. La historia debe ser convincente y clara pero compleja e incrustada en un campo académico.

\section{Resultados}

Las entrevistas se transcribieron para su respectivo análisis, se leyeron repetidamente. Para complementar la familiarización con los datos se realizó una tabla en el programa de Microsoft Excel en la que se escribieron los argumentos y procedimientos empleados por cada estudiante en cada una de las tareas planteadas. Se resaltaron las frases más significativas o claves que refieren a la pendiente y se 
denominaron como códigos iniciales, dando lugar así a la segunda fase del análisis temático. En este escrito, sólo se presentan algunos resultados de las codificaciones iniciales. Por ejemplo, la tarea 1 consistió en asociar imágenes que tuvieran relación con la pendiente, con el objetivo de conocer su idea general de pendiente, además de identificar si pesa más las situaciones escolares o las de su vida cotidiana. Se dieron cuatro imágenes: una cancha de futbol, una recta presentada en el plano cartesiano, la gráfica que representa la distancia recorrida en función del tiempo en el lanzamiento de un balón y por último un cerro. En esta tarea se identificaron quince códigos (palabras en cursivas) de los cuales fue posible agrupar en cuatro categorías tal como se muestra en la tabla 1. Algunos códigos no se presentan dado que no aparecen tan frecuentes, pero si serán considerados en el resto del análisis.

Tabla 1. Codificaciones iniciales correspondientes a la tarea 1

\begin{tabular}{|l|l|l|}
\hline Categoría & \multicolumn{1}{|c|}{ Códigos } & \multicolumn{1}{c|}{ Ejemplo de extracto de la entrevista } \\
\hline Recta & $\begin{array}{l}\text { Diagonal } \\
\text { Segmento } \\
\text { Línea recta }\end{array}$ & $\begin{array}{l}\text { E17: Una pendiente es digámoslo como una línea } \\
\text { diagonal. } \\
\text { E6: Aquí vemos una recta que está inclinada y tiene } \\
\text { un ángulo menor a 90 y la pendiente es la recta. }\end{array}$ \\
\hline $\begin{array}{l}\text { Subida } \\
\text { bajada }\end{array}$ & $\begin{array}{l}\text { Caída o bajada } \\
\text { Va decayendo }\end{array}$ & $\begin{array}{l}\text { E5: La pendiente es algo así como una caída, como } \\
\text { que va de bajada [simula con brazo una diagonal]. } \\
\text { E17: Una pendiente es como una línea por así decirlo, } \\
\text { es como una caída o subida por donde va a subir } \\
\text { cierto objeto o va a bajar cierto objeto. }\end{array}$ \\
\hline Inclinación & $\begin{array}{l}\text { Tiene } \\
\text { inclinación } \\
\text { Forma } \\
\text { ángulo }\end{array}$ & $\begin{array}{l}\text { E21: La montaña tiene un punto más alto y un punto } \\
\text { bajo y tiene una inclinación. } \\
\text { E8: La b también tiene una inclinación. }\end{array}$ \\
\hline Distancia & $\begin{array}{l}\text { Es una medida } \\
\text { Es un recorrido }\end{array}$ & $\begin{array}{l}\text { E15: La pendiente es como una medida y aquí mediría } \\
\text { esto [señala la recta] } \\
\text { E7: La b está midiendo una cierta distancia de dónde } \\
\text { avanza el objeto. }\end{array}$ \\
\hline
\end{tabular}

En algunos extractos de las entrevistas aparece más de un código, de manera que se consideran de forma separada. En esta tarea, el código recta o diagonal es identificado en al menos $40 \%$ de los estudiantes quienes hacen explícito que la pendiente es la recta, otros estudiantes complementan con características como: está en forma de diagonal, tiene una elevación, tiene una inclinación, etc. La imagen que asocian más con relación a la pendiente es la recta en el plano cartesiano (75\%) y la representación del lanzamiento de un balón (53\%). Un 34\% de los estudiantes asocia con la imagen del cerro y un 12\% con la cancha de futbol. Cabe mencionar que entre los argumentos se destaca que la elección de la cancha de futbol se debe a que piensan en el lanzamiento de la pelota, es decir en la actividad que se realiza en ella. 
En otra tarea, la dos, se pide la pendiente de una calle que tiene que recorrer un auto para llegar a una cima. La imagen empleada para representar la situación vislumbra un triángulo rectángulo con sus correspondientes medidas (Figura 1).

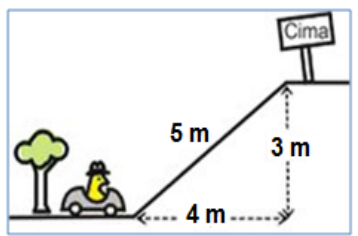

Figura 1. Imagen mostrada para la tarea 2

Para esta tarea se encontraron 14 códigos y por el momento se han agrupado en tres categorías, tal como se muestra en la tabla 2.

\begin{tabular}{|l|l|l|}
\hline Categoría & \multicolumn{1}{|c|}{ Códigos } & \multicolumn{1}{c|}{ Ejemplo de extracto de la entrevista } \\
\hline Distancia & $\begin{array}{l}\text { Distancia } \\
\text { recorrer } \\
\text { Distancia mínima } \\
\text { Medida } \\
\text { Altura }\end{array}$ & $\begin{array}{l}\text { E3: Es la distancia que tiene que recorrer de este } \\
\text { punto a este punto. } \\
\text { E8: Por como está representada es como la mejor } \\
\text { vía para llegar acá arriba. }\end{array}$ \\
\hline Inclinación & $\begin{array}{l}\text { Inclinación } \\
\text { Diagonal }\end{array}$ & $\begin{array}{l}\text { E2: Sería la diagonal que son } 5 \text { metros } \\
\text { E8: La pendiente vendría siendo esa línea, la } \\
\text { diagonal. }\end{array}$ \\
\hline $\begin{array}{l}\text { Subida } \\
\text { bajada }\end{array}$ & $\begin{array}{l}\text { Subida } \\
\text { Descenso }\end{array}$ & $\begin{array}{l}\text { E19: No tiene pendiente porque no hay un } \\
\text { descenso. } \\
\text { E22: Porque esa es una pendiente en sí [señala la } \\
\text { hipotenusa], todo lo que valla hacia arriba y } \\
\text { hacia abajo se conoce como pendiente. }\end{array}$ \\
\hline
\end{tabular}

Tabla 2. Codificaciones iniciales correspondientes a la tarea 2

Esta tarea se planteó con el objetivo de mirar que empleaban para calcular la pendiente y si se mantenía la idea de la pendiente como el segmento o la diagonal. Como resultado de la primera codificación a esta tarea se encontró que la idea de pendiente es asociada explícitamente con la distancia, ya sea distancia por recorrer o distancia mínima en al menos un 50\% de los estudiantes, otros códigos asociados a esa categoría es la medida de la altura o de la distancia horizontal. Un $25 \%$ de los estudiantes esgrime explícitamente la pendiente como la recta y un $24 \%$ con la propiedad de tener una inclinación, ser una subida o una bajada. Con el objetivo de confirmar la idea de pendiente como la distancia o longitud, se planteó una tercera tarea en la que se dan tres techos con la misma pendiente pero de diversos tamaños y se preguntó sobre el techo que tuviera mayor pendiente. En esta tarea de confirmó la idea de pendiente como longitud 
en al menos un $72 \%$ de los estudiantes, quienes justificaron su decisión atribuida al tamaño de los techos y dejando de lado la inclinación. El resto de los estudiantes decide por la inclinación de los techos, es decir confirman que los techos tienen la misma pendiente.

\section{Reflexiones finales}

En las principales concepciones detectadas en los estudiantes se muestra la idea de la pendiente como la recta, como una distancia y como la inclinación. Esta última en un sentido físico es decir, como al desnivel de un terreno, atribuido a ejemplos de subidas o bajadas. Cuando se les pidió a los estudiantes relacionar la pendiente con situaciones escolares o con situaciones de la vida real, notamos que la asocian con la gráfica de una recta y con escasa frecuencia con situaciones de la vida real. Sin embargo, en los argumentos de los estudiantes figura su experiencia en situaciones de su vida cotidiana, como lo es con lo empinado o inclinado, pero al momento de enfrentarse a las tareas que se les plantearon pesó más sus experiencias escolares. ¿A qué se debe que omitan sus concepciones asociadas a su experiencia en la vida real?, ¿De qué manera se puede introducir a la pendiente considerando las concepciones que se encuentren en estos estudiantes? En este momento no estamos en condiciones de responder a estas preguntas, sin embargo podemos notar que su enseñanza se ha centrado sólo en lo procedimental y en el contexto matemático, dejando de lado su relación con otros contextos. Esta enseñanza conlleva al estudiante a formarse la idea de que lo que aprende en sus clases de matemáticas sólo le va a servir para aprobar sus cursos de matemáticas, orillando la categorización de sus aprendizajes por asignaturas y sin relación entre sí.

\section{Referencias Bibliográficas}

Ausubel, D. (1978). Psicología Educativa. Un punto de vista cognitivo. México: Trillas.

Braun, V., \& Clarke, V. (2012). Thematic analysis. In H. Cooper (Ed.), APA Handbook of research methods in psychology: Research Designs (pp: 57-71). Washington (DC): American Psychological Association.

Casey, S., \& Nagle, C. (2016). Students' use of slope conceptualizations when reasoning about the line of best fit. Educational Studies in Mathematics, 92(2), 163-177.

Dolores, C. (2013). La variación y la derivada. México, D. F.: Díaz de Santos.

Dolores, C., Alarcón, G., y Albarrán, D. (2002). Concepciones alternativas sobre las gráficas cartesianas del movimiento: el caso de la velocidad y la trayectoria. RELIME. Revista latinoamericana de investigación en matemática educativa, 5(3), 225-250.

Herbert, S., y Pierce, R. (2008). An 'Emergent Model' for Rate of Change. International Journal of Computers for Mathematical Learning, 13(3), 231-49.

Hoffman, W. (2015). Concept image of slope: Understanding middle school mathematics teachers' perspective through task-based interviews (Doctoral dissertation) The University Of North Carolina At Charlotte.

Goldin, G. (1997). Chapter 4: Observing Mathematical Problem Solving through Task-Based Interviews. Journal for Research in Mathematics Education. Monograph, 9, 40-177. 
Moore-Russo, D., Conner, A., y Rugg, K. (2011). Can slope be negative in 3-space? Studying concept image of slope through collective definition construction. Educational Studies in Mathematics, 76(1), 3-21.

Nagle, C., y Moore-Russo, D. (2014). Slope across the Curriculum: Principles and Standards for School Mathematics and Common Core State Standards. Mathematics Educator, 23(2), 40-59.

Nagle, C., Moore-Russo, D., Viglietti, J., y Martin, K. (2013). Calculus students'and instructors'conceptualizations of slope: a comparison across academic levels. International Journal of Science and Mathematics Education, 11(6), 1491-1515.

Nájera, J. (2015). Conexiones que establecen los estudiantes de bachillerato al resolver Problemas de razones de cambio. Tesis de maestría no publicada. Universidad Autónoma de Guerrero, México.

Planinic, M., Milin-Sipus, Z., Katic, H., Susac, A., y Ivanjek, L. (2012). Comparison of student understanding of line graph slope in physics and mathematics. International journal of science and mathematics education, 10(6), 1393-1414.

SEP, (2011), Programas de estudio 2011 guía para el maestro. Educación secundaria. Matemáticas. México, D. F.: Secretaría Educación Pública. Consultado http://evaluaciondocente.sep.gob.mx/materiales/SEPPROGRAMASDEESTUDIO2011.GUIAPARAELMAE STRO.SECUNDARIA.MATEMATICAS.pdf

Stanton, M., y Moore- Russo, D. (2012). Conceptualizations of slope: A review of state standards. School Science and Mathematics, 112(5), 270-277.

Stump, S. (1999). Secondary mathematics teachers' knowledge of slope. Mathematics Education Research Journal, $11(2), 124-144$.

Stump, S. (2001). High school precalculus students' understanding of slope as measure. School Science and Mathematics, 101(2), 81-89.

Teuscher, D., y Reys, R. (2010). Slope, rate of change, and Steepness: Do students understand the concepts? Mathematics Teacher, 3(7), 519-524

Teuscher, D., y Reys, R. (2012). Rate of Change: AP Calculus Students' Understandings and Misconceptions after Completing Different Curricular Paths. School Science and Mathematics, 112(6), 359-376.

Thompson, A. G. (1992). Teachers' beliefs and conceptions: A synthesis of the research. In D. A. Grouws (Ed.), Handbook of research on mathematics teaching and learning (pp. 127-146). Reston, VA: National Council of Teachers of Mathematics.

Walter, J. G., y Gerson, H. (2007). Teachers' personal agency: Making sense of slope through additive structures. Educational Studies in Mathematics, 65(2), 203-233.

Wagener, L. (2009). A Worthwhile Task to Teach Slope. Mathematics Teaching in the Middle School, 15(3), 168174.

Wilhelm, J., y Confrey, J. (2003). Projecting Rate of Change in the Context of Motion onto the Context of Money. International Journal of Mathematical Education in Science and Technology, 34(6), 887-904. 